\title{
Flux Sensitivity of VERITAS
}

\author{
V. V. Vassiliev \\ Whipple Observatory, Harvard-Smithsonian CfA, P.O. Box 97, Amado, AZ \\ 85645, USA \\ For the VERITAS Collaboration
}

\begin{abstract}
VERITAS is a new major ground-based gamma-ray observatory with an array of seven $10 \mathrm{~m}$ optical reflectors to be built at the Whipple Observatory in southern Arizona, USA. It will consist of an array of imaging Cherenkov telescopes designed to conduct critical measurements of AGNs and SNRs in the energy range of $50 \mathrm{GeV}$ - $50 \mathrm{TeV}$. The design of the array has been optimized for the highest sensitivity to point sources in the $100 \mathrm{GeV}-10 \mathrm{TeV}$ band when the stereoscopic imaging technique is employed. Maximum versatility of the array has been another major optimization criterion. We present the flux sensitivity of the baseline VERITAS configuration.
\end{abstract}

\section{Introduction}

A new generation of very high energy ground based $\gamma$-ray observatories (VERITAS [1], HESS [2], MAGIC [3]) promises to extend their sensitive energy range to below $100 \mathrm{GeV}$, an energy band which is expected to contain a wealth of new information on high energy physics and astrophysics (for review see [4]). The spectra and variability of AGNs, the origin of high energy cosmic rays, physical processes in strong magnetic fields of pulsars, the puzzle of dark matter, the type of cosmology of the Universe, origin of $\gamma$ ray bursts, evaporation of black holes, and even a quantum gravity observable effects, are all in the scope of phenomena which will be studied by these projects. In this paper we give the technical characteristics of VERITAS driven by scientific goals. Also, the main motivations for choices of the array parameters are explained. Finally, we discuss factors which limit the flux sensitivity. 


\section{Simulations}

To predict the performance of VERITAS, the response of the array has been simulated with the use of the DePauw-Purdue KASCADE system of air shower Monte Carlo (MC) programs [5]. The study of hadronic showers which may mimic pure electromagnetic cascades has been done with the CORSIKA code [6]. To determine the optimum VERITAS design we have studied the effects of varying the number of telescopes in an array, the spacing between them, reflector aperture, telescope focal length, camera Field of View (FoV) and pixel size. The characteristics of the baseline configuration as well as simulation input parameters are summarized in Table 1.

Table 1

Specifications of the baseline VERITAS design.

\begin{tabular}{|c|c|}
\hline Location & Montosa Canyon, Arizona, USA \\
\hline Array elevation & $1390 \mathrm{~m}$ a.s.l. \\
\hline Number of telescopes & 7 (hexagonal layout) \\
\hline Telescope spacing & $80 \mathrm{~m}$ \\
\hline Mirror & Davies-Cotton \\
\hline Reflector aperture/area & $10 \mathrm{~m} / 78.6 \mathrm{~m}^{2}$ \\
\hline Focal length & $12 \mathrm{~m}$ \\
\hline Facets & $244,61 \mathrm{~cm}$ hexagon \\
\hline Camera & Homogeneous \\
\hline Field of View & $3.5 \mathrm{deg}$ \\
\hline Number of pixels & 499 \\
\hline Pixel Spacing/Photocathode Size & $0.148 \mathrm{deg} / 0.119 \mathrm{deg}$ \\
\hline ADC integration gate & $8 \mathrm{nsec}$ \\
\hline Pixel BS noise & 1.1 pe pixel $^{-1}$ \\
\hline Weathering reflectivity factor & 0.9 \\
\hline Light-concentrator enhancement & 1.35 \\
\hline Telescope Triggers & 2,3 pixels (adjacent) \\
\hline Pixel coincidence gate & 15 nsec \\
\hline Array Trigger & 3 telescopes out of 7 \\
\hline Telescope coincidence gate & $40 \mathrm{nsec}$ \\
\hline
\end{tabular}

\section{VERITAS design}

The VERITAS design has been optimized for maximum sensitivity to point sources in the energy range $100 \mathrm{GeV}-10 \mathrm{TeV}$, but with significant sensitivity in the range $50 \mathrm{GeV}-100 \mathrm{GeV}$ and from $10 \mathrm{TeV}$ to $50 \mathrm{TeV}$. Optimization has been performed with fixed total number of channels. The details of the 
VERITAS design study are given in [8]. Here we summarize our arguments for the baseline configuration.

- VERITAS should have at least $6-7$ telescopes for good wide energy range sensitivity and versatility. Seven telescopes provide more flexibility in VERITAS operation modes when the array is split into sub-arrays. The array of three $15 \mathrm{~m}$ telescopes is rejected because of poor performance at high energies and lack of versatility.

- Camera FoV, $3.5^{\circ}$, is a compromise between achieving low energy threshold of the array and array performance at high energies, and the ability to conduct an efficient sky survey and study extended sources. Also, the chosen $\mathrm{FoV}$ is the minimum necessary for effective image reconstruction with a single telescope.

- The given FoV together with the number of channels per telescope, 500, translates into a pixel spacing of $0.149^{\circ}$.

- The optical system of the telescope is proposed to be $f / 1.2$. This provides an adequate match to the number of channels in the telescope camera making reflector global aberrations at the edge of the FoV comparable to the pixel size. The $f / 1.5$ system would perform better but it would require a substantial additional investment in an optical support structure and system of mirror alignment.

- The telescope aperture, $10 \mathrm{~m}$, is chosen by previous successful experience of operating the Whipple Observatory telescope and for economy.

- Spacing between telescopes should be in the range 70-80 m. Decrease of the spacing reduces efficient event reconstruction, background rejection and array sensitivity in the range $200 \mathrm{GeV}-1 \mathrm{TeV}$. Increasing the spacing, does not change array sensitivity in this energy range, but it increases the array energy threshold.

\section{VERITAS flux sensitivity}

The VERITAS flux sensitivity has been estimated for 50 hours of observations on a point source with a spectrum given by $d N_{\gamma} / d E \propto E^{-2.5}$ as is seen from the Crab Nebula in the sub-TeV energy range [7]. The minimum detectable flux of $\gamma$-rays is constrained by the $5 \sigma$ confidence level or by the statistics of the detected photons, $N_{\gamma}>10$, when the background is almost negligible. The details of the sensitivity calculations can be found in [8].

The VERITAS $\gamma$-ray flux sensitivity as a function of array energy threshold is shown in Figure 1. At high energies, above $\sim 2-3 \mathrm{TeV}$, VERITAS sensitivity is limited by photon statistics. In this region sensitivity is depressed with energy increase due to a limited FoV of the telescope camera. Large zenith angle observations can improve array performance in this energy band. In the 


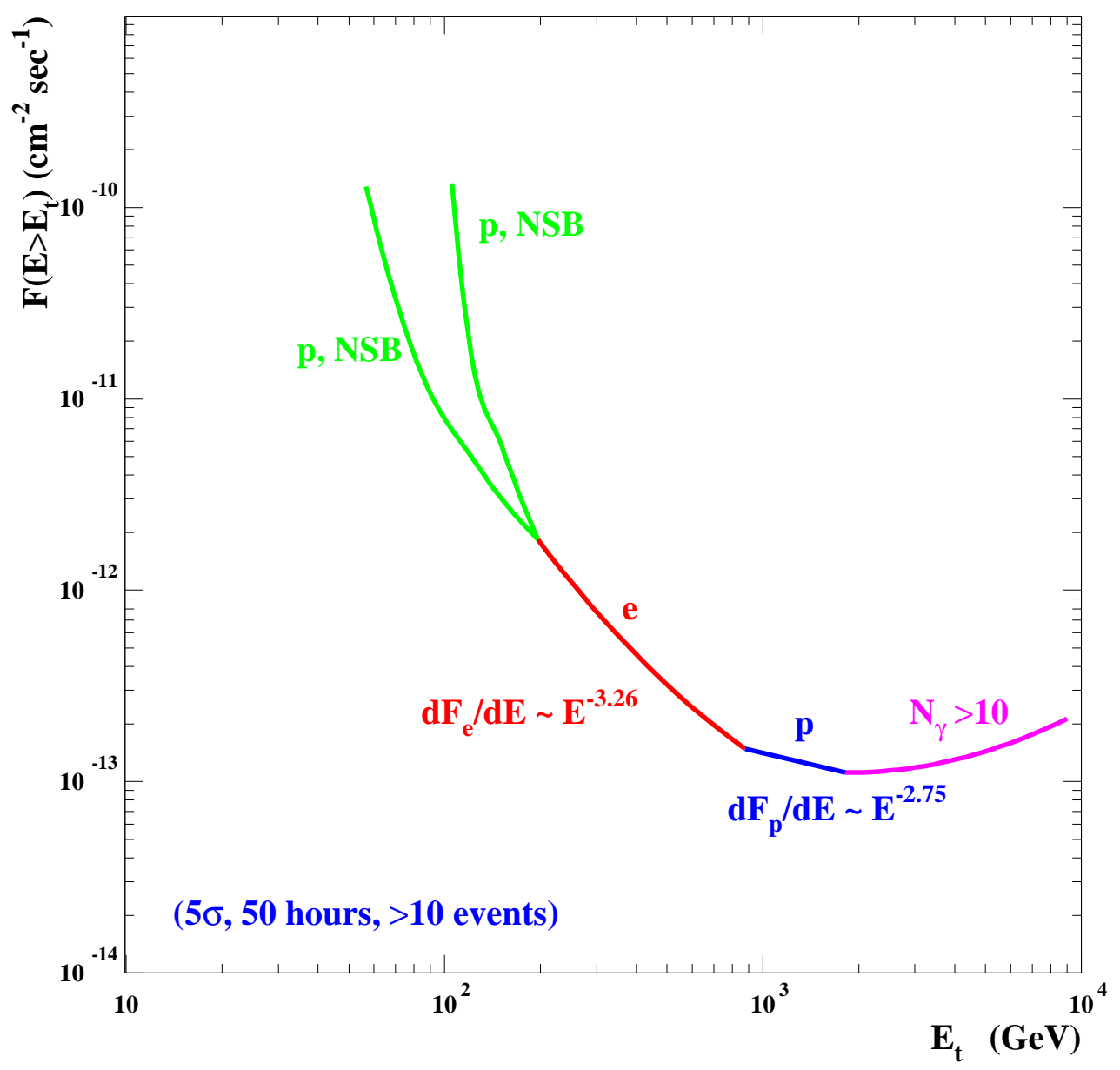

Fig. 1. The sensitivity of VERITAS to a point-like source in 50 hours of observing. The dominant background for VERITAS as a function of energy threshold is indicated (see text for details).

vicinity of $1 \mathrm{TeV}$, VERITAS sensitivity will likely be limited by cosmic ray (CR) protons which mimic $\gamma$-ray showers. The detection rate of this isotropic background is not well known and it may be of scientific interest by itself because of the large uncertainty in its predicted effect by different proton interaction models available for study within the CORSIKA code. The energy region from $200 \mathrm{GeV}$ to $\sim 1 \mathrm{TeV}$ will most likely be dominated by the diffuse electron background. The steepness of the CR electron spectrum causes this to become a limiting factor of the VERITAS sensitivity. The region below $\sim 200$ $\mathrm{GeV}$ is strongly affected by the night sky background (NSB) and CR protons. Two curves in this region show the difference in array sensitivity depending on the conditions of the observations. In a favorable situation, dark observation field, the array energy threshold for events which we are able to reconstruct may decrease to as low as $40-50 \mathrm{GeV}$. The single telescope accidental trigger 
rate $(<0.1-1.0 \mathrm{MHz})$, however, may limit array operation energy threshold to $\sim 70 \mathrm{GeV}$. If observations are carried out in a bright region of sky (Milky Way, lower elevation) where the NSB is $\sim 4$ times brighter, VERITAS may be limited to a $110 \mathrm{GeV}$ energy threshold. The result of observations in this energy band will be highly sensitive to array trigger condition, and event reconstruction and background rejection methods. The plot is indicative of our current achievement, which will certainly be improved.

\section{References}

[1] VERITAS "Letter of Intent" (1998)

[2] W. Hofmann, Proc. Workshop on TeV $\gamma$-ray Astropys., South Africa, Ed. O.C. de Jager (1997) 405.

[3] J.A. Barrio, et al. "The Magic Telescope", design study, MPI-PhE/98-5 (1998).

[4] T. Weekes, Nobel Symposium "Particle Physics and the Universe" (August 1998), Physica Scripta in press.

[5] M.P. Kertzman \& G.H. Sembroski, Nucl. Inst. Meth. A, 343 (1994) 629.

[6] D. Heck, et al., Forschungszentrum Karlsruhe Report FZKA 6019 (1998).

[7] A.M. Hillas, et al. Astrophys. J. 503 (1998) 744.

[8] T. Weekes, et al. VERITAS proposal (1999) in preparation. 